\title{
Effect of a Novel Thermostatic Device on Meibomian Gland Dysfunction: A Randomized Controlled Trial in Chinese Patients
}

\author{
Siyuan Li $\cdot$ Ke Yang $\cdot$ Jingyi Wang $\cdot$ Shang Li $\cdot$ Lei Zhu \\ Jun Feng $\cdot$ Lei Tian · Ying Jie (D)
}

Received: August 17, 2021 / Accepted: November 10, 2021 / Published online: November 25, 2021

(c) The Author(s) 2021

\begin{abstract}
Introduction: Meibomian gland dysfunction (MGD) is a common eye condition that causes excessive evaporation of tears by changing the tear film composition. Current treatments often fail to produce satisfactory results, which is mostly due to poor patient adherence. This study aimed to evaluate the effectiveness and safety of the MiBoFlo Thermoflo ${ }^{\circledR}$ on both subjective symptoms and objective signs in Chinese patients with MGD.

Methods: This prospective, randomized, controlled clinical trial included 108 eyes of 54 patients with MGD who were recruited in Beijing Tongren Hospital and randomized 1:1 to MiBoFlo ( $n=54$ eyes) or LipiFlow ${ }^{\circledR} \quad(n=54$ eyes) treatment group. In the MiBoFlo group, patients received three 10-min treatments, each spaced 2 weeks apart, and the treatment was
\end{abstract}

Siyuan Li and Ke Yang contributed equally as co-first authors.

Ying Jie and Lei Tian contributed equally as cocorresponding authors.

S. Li · K. Yang · J. Wang · S. Li - L. Zhu · J. Feng ·

L. Tian · Y. Jie (\)

Beijing Institute of Ophthalmology, Beijing

Tongren Eye Center, Beijing Tongren Hospital, Capital Medical University, Beijing Ophthalmology and Visual Science Key Lab, No. 1 Dong Jiao Min Xiang, Dong Cheng District, Beijing 100730, China e-mail: jie_yingcn@aliyun.com followed by eyelid compression each time. Patients in the LipiFlow group received a single 12-min treatment. The primary parameters measured included changes in Ocular Surface Disease Index (OSDI) score, Meibomian Glands Yielding Liquid Secretion (MGYLS) score, and Meibomian Glands Secretion (MGS) score from baseline to 2 months. The secondary parameters included tear meniscus height (TMH), non-invasive keratograph break-up time (NIKBUT), corneal fluorescein staining (CFS), and meibomian glands (MG) loss from baseline to 2 months. Safety parameters include visual acuity (VA), intraocular pressure (IOP), anterior segment, and facial skin.

Results: The OSDI, MGYLS, and MGS scores all improved from baseline to 1 month in both MiBoFlo and LipiFlow groups, and these improvements were maintained at 2 months. CFS score, NIKBUT, and MG loss showed no significant change in both groups.

Conclusion: As a portable and comfortable device, MiBoFlo can improve the treatment of MGD and achieve a sustained improvement in both symptoms and meibomian gland function lasting at least 2 months.

Keywords: MiBoFlo; LipiFlow; MGD; Dry eye; Therapy

Abbreviations

CFS Corneal fluorescein staining 


$\begin{array}{ll}\text { IOP } & \text { Intraocular pressure } \\ \text { MG } & \text { Meibomian glands } \\ \text { MGD } & \text { Meibomian gland dysfunction } \\ \text { MGS } & \begin{array}{l}\text { Meibomian Glands Secretion } \\ \text { MGYLS }\end{array} \\ & \begin{array}{l}\text { Meibomian Glands Yielding Liquid } \\ \text { Secretion }\end{array} \\ \text { NIKBUT } & \begin{array}{l}\text { Non-invasive keratograph break-up } \\ \text { time }\end{array} \\ \text { OSDI } & \text { Ocular Surface Disease Index } \\ \text { TBUT } & \text { Tear film break-up time } \\ \text { TMH } & \text { Tear meniscus height } \\ \text { VA } & \text { Visual acuity }\end{array}$

\section{Key Summary Points}

Meibomian gland dysfunction has become a high-incidence disease, affecting people's quality of life ever more seriously.

Physical therapy is the basis of meibomian gland dysfunction treatment, and MiBoFlo Thermoflo ${ }^{\circledR}$ is a new therapeutic device which can transfer heat to the eyelid at constant temperature.

We aim to evaluate the effectiveness and safety of MiBoFlo therapy on both subjective symptoms and objective signs of meibomian gland dysfunction patients in China.

MiBoFlo therapy improves both symptoms and MGD compared to current treatment methods that can last up to 2 months.

\section{INTRODUCTION}

As a chronic disease, dry eye disease (DED) is no longer just a condition affecting the elderly. Current prevalence in teenagers has been steadily increasing on a yearly basis $[1,2]$. Meibomian gland dysfunction (MGD), which causes excessive evaporation of tears by changing the tear film composition, is considered a leading cause of DED [3-5].
Characterized by the meibomian gland obstruction, MGD is currently managed by two main types of treatments, i.e., pharmacotherapy and physical therapy [6]. It has been shown that warm compresses are an effective way to relieve eye discomfort [4]; however, patient adherence is often poor because of the requirement of time and the difficulty maintaining the temperature of the warm compresses for an extended period of time. In addition to this, the temperature of warm compresses is not enough to completely melt the obstructive material within the meibomian gland excretory duct [7]. Over recent years, the LipiFlow Thermal Pulsation System ${ }^{\circledR}$ (TearScience, Morrisville, NC, USA) has emerged as a new type of MGD therapy device, which clears the blocked meibomian glands by simultaneously applying controlled heat and graded pulsatile pressure to the outer and inner surface of the eyelids [8]. It was first described by Korb and Blackie in 2010 [9], and afterward its clinical effects have been proved by a number of studies [10].

MiBoFlo Thermoflo ${ }^{\circledR}$ (Mibo Medical, Dallas, TX, USA) is a new thermostatic device for MGD treatment that is composed of a small embedded computer and a handheld probe connected to it. It applies heat thermoelectrically through a silver pressure plate to the outer eyelid, whilst maintaining the temperature constant at $42{ }^{\circ} \mathrm{C}$ [11]. While using the device, the practitioner simultaneously administers manual massage by reciprocating and rotating the probe. Currently, there are few reports on MiBoFlo; Kenrick and Alloo measured the palpebral conjunctiva temperature before and after MiBoFlo therapy using a non-contact infrared thermometer; however, they did not report its clinical effects [12].

Thus, the aim of our randomized, prospective, preliminary clinical study was to evaluate the effectiveness and safety of treatment with the MiBoFlo on both subjective symptoms and objective signs of patients with MGD in China. 


\section{METHODS}

\section{Study Design}

This prospective, randomized, observer-masked clinical trial aimed to evaluate the effectiveness and safety of treatment with the MiBoFlo on both subjective symptoms and objective signs of patients with MGD, comparing the therapeutic effects, superiority, and differences between the MiBoFlo and the LipiFlow therapy. The study was conducted according to the criteria set by the declaration of Helsinki and each subject signed informed consent before participating in the study. The study protocol was approved by the Ethics Review Committee of Beijing Tongren Hospital. The trial was registered at www.clinicaltrials.gov as NCT04310969 first posted on 17 March 2020.

\section{Study Population}

Patients who met the following inclusion criterion were included: 18 years of age or older; meet the diagnostic criteria for dry eye developed by DEWS II: Ocular Surface Disease Index (OSDI) score $\geq 13$ points, tear film break-up time (TBUT) $<10 \mathrm{~s}$; meet the signs of MGD: the presence of lid margin abnormalities, orifice abnormalities, and meibum abnormalities.

Patients with one of the following conditions were excluded from the group: used artificial tears other than aqueous supplementation; used non-steroidal anti-inflammatory drugs, topical glucocorticoids, immunomodulators within 1 month; had skin allergies or inflammation; had a history of ocular surgery, eyelid surgery, or neurological paralysis within 6 months; had active ocular infection or inflammation; had a history of systemic disease affecting ocular surface function, such as Stevens-Johnson syndrome, Sjögren syndrome, etc.

\section{Treatment}

A total of 54 subjects (108 eyes) who met inclusion criteria in clinics at Beijing Tongren Hospital were enrolled for this study in June
2020. The MiBoFlo treatment group was set as an experimental group, and the LipiFlow group was set as a positive control group. The subjects were randomized 1:1 to the MiBoFlo treatment group (54 eyes) and LipiFlow treatment group (54 eyes) using a randomized digital table by the randomization manager.

In the MiBoFlo group, patients received three 10-min treatments, each spaced 2 weeks apart. MiboFlo therapy was instantly followed by eyelid compression. Patients in the LipiFlow group received a single 12-min treatment without any other physical therapy. Medications before and after treatment in both groups remained unchanged. Eyedrops mentioned in exclusion criteria were not allowed to be used during the study.

In the MiBoFlo group, we first used the security key to start and preheat the machine to $42.5^{\circ} \mathrm{C}$. Patients were required to stay in the supine position. Both eyelids were cleaned and smeared with a small amount of ultrasound gel in order to reduce friction between the device and eyelid skin. Next, we massaged the outer skin of the upper and lower eyelids by reciprocating and rotating the handheld probe for a period of $10 \mathrm{~min}$. The same was done for the other eye. Eyes were closed during therapy, and eyelid compression was instantly applied following therapy. Two wet cotton swabs squeezed the eyelids, one on the inner surface of the eyelid and the other one on the outer lid, to apply force to express the liquefied obstructive material.

The treatment of the LipiFlow group was performed on the same day as the first treatment of patients in the MiBoFlo group. Patients were required to stay in the Fowler position and were administered a drop of tropical anesthetic in both eyes. A sterile eye cup was placed on to the conjunctival sac and fixed to the skin with surgical tape. Next, the program started heating, applying constant pressure, increasing pressure, and eventually alternating pressure. After 12-min treatment, eye cups were slightly removed from both eyes. 


\section{Effectiveness Parameters}

The evaluation data of each patient before the first treatment were used as the baseline. The follow-up examinations were scheduled at 1 month and 2 months after the first treatment. The evaluator was blinded to the group allocations. The parameters used to evaluate the effectiveness and safety of both treatment groups are described below.

\section{Primary Parameters}

The OSDI questionnaire was chosen to assess subjective symptoms of dry eye, which can demonstrate sensitivity and specificity in distinguishing between normal subjects and patients with dry eye disease. The overall OSDI score defined the ocular surface as normal (0-12 points), mild (13-22 points), moderate (23-32 points), or severe (33-100 points) [13].

Meibomian Glands Yielding Liquid Secretion (MGYLS) was standardized using the Meibomian Gland Evaluator. A total of 30 glands were evaluated along the eyelid margin, consisting of five consecutive glands located in each of the nasal, central, and temporal regions of the upper and lower eyelids, respectively. For each of five consecutive glands, MGYLS was scored using the following grading system: 0 , which indicated all five glands have secretory capacity; 1, which indicated 3-4 glands have secretory capacity; 2, which indicated 1-2 gland(s) has(have) secretory capacity; 3 , which indicated no gland has secretory capacity. The total MGYLS score (range 0-18) was calculated as the sum of the grades for six areas of each eye.

Meibomian Glands Secretion (MGS) was standardized using the Meibomian Gland Evaluator. For each of five consecutive glands, MGS was scored using the following grading system: 0 , which indicated clear liquid secretion; 1 , which indicated cloudy liquid secretion; 2, which indicated granular secretion; 3, which indicated toothpaste-like opaque secretion.

\section{Secondary Parameters}

The lower eyelid tear meniscus was photographed under a white light source by Oculus Keratograph 5M® (Oculus Optikgeräte $\mathrm{GmbH}$,
Wentzler, Germany), and tear meniscus height (TMH) was measured with the built-in ruler in order to estimate tear secretion.

The non-invasive keratograph break-up time (NIKBUT) was used to evaluate tear film stability using Oculus Keratograph 5M. The average NIKBUT was recorded into the statistical result.

Fluorescein was applied in the lower conjunctival sac of each eye with a fluorescein sodium ophthalmic strip (Jingming New Technological Development Co., Ltd, Tianjin, China), and corneal erosion assessed by corneal fluorescein staining (CFS) was scored using the following grading system: grade 0 , which indicated no corneal erosion; grade 1 , which indicated 1-5 punctate epithelial erosions seen inferiorly; grade 2 , which indicated $6-30$ punctate epithelial erosions; grade 3, which indicated more than 30 punctate epithelial erosions [14].

Meibography was performed using MeiboScan attached to the Oculus Keratograph 5M. Structural changes of meibomian were observed with the infrared light source. The area of MG loss was measured with ImageJ software, and its relation to the total area was noted as a percentage [15].

\section{Safety Index}

Visual acuity (VA), intraocular pressure (IOP), anterior segment, and facial skin were observed at every follow-up so as to ensure the safety of treatment.

\section{Statistical Analysis}

Statistical analysis was performed using SPSS software (IBM Corp, Somers, NY) and GraphPad Prism version 9. Independent-samples $t$ test and Mann-Whitney $U$ test were used to assess the baseline of the evaluation parameters. Two-way analysis of variance (ANOVA) was used to assess the effects of the MiBoFlo and LipiFlow treatment on the primary and secondary parameters assessed at baseline, 1 month, and 2 months. A statistically significant difference was based on the level $\alpha=0.05$. 
Table 1 Baseline evaluation parameters

\begin{tabular}{lllllll}
\hline & MiboFlo & & & \multicolumn{2}{l}{ LipiFlow } & \multirow{2}{*}{$\boldsymbol{p}$} \\
\cline { 2 - 3 } & Number & Mean \pm SD/median & & Number & Mean \pm SD/median & \\
\hline OSDI score & 22 & $44.31 \pm 19.03$ & 20 & $46.09 \pm 16.70$ & $0.75^{\mathrm{a}}$ \\
MGYLS score & 44 & 9.5 & 40 & 9 & $0.80^{\mathrm{b}}$ \\
MGS score & 44 & 8 & 40 & 11 & $0.04^{\mathrm{b}}$ \\
CFS score & 44 & 0 & 40 & 0 & $0.07^{\mathrm{b}}$ \\
TMH (mm) & 44 & $0.23 \pm 0.07$ & 40 & $0.25 \pm 0.12$ & $0.36^{\mathrm{a}}$ \\
NIKBUT (s) & 44 & $6.44 \pm 3.46$ & 40 & $5.78 \pm 2.95$ & $0.35^{\mathrm{a}}$ \\
Area of MG loss & 44 & $0.27 \pm 0.17$ & 40 & $0.22 \pm 0.11$ & $0.07^{\mathrm{a}}$ \\
\hline
\end{tabular}

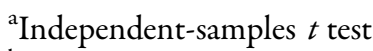

${ }^{\mathrm{b}}$ Mann-Whitney $U$ test

We estimated that the primary parameters would have to be analyzed in 44 subjects ( 22 subjects in each group) to achieve the study power of at least $80 \%$ for a significant $P$ value of 0.05 with the two-tailed test, assuming the dropout rate of $20 \%$ [16].

\section{RESULTS}

A total of 54 subjects (108 eyes), aged 22-78 years were enrolled. In the end, there were 22 subjects in the MiBoFlo group who completed the final follow-up and 20 subjects in the LipiFlow group in total. The MiBoFlo group included 17 women and five men with an average age of $43.95( \pm 11.40)$ years. The LipiFlow group included 15 females and five males with an average age of $41.90( \pm 11.03)$ years.

The baseline values of each evaluation parameter of the two devices were compared before the treatment. No significant difference was found at baseline levels of OSDI score, MGYLS score, CFS score, NIKBUT, TMH, and area of MG loss in both groups. However, a significant intergroup difference was found in the baseline level of the MGS score (Table 1).

The symptoms of patients with dry eye were assessed by OSDI questionnaire. In the MiBoFlo group, the OSDI score decreased from $44.31 \pm 1.03$ at baseline to $34.00 \pm 13.84$ at 1 month $(p<0.01)$, and to $28.65 \pm 18.20$ at
2 months $(p<0.0001)$. In the LipiFlow group, the OSDI score decreased from $46.10 \pm 17.70$ at baseline to $33.28 \pm 21.41$ at 1 month $(p<0.001)$, and to $30.63 \pm 19.86$ at 2 months $(p<0.0001) \quad$ (Fig. 1). OSDI improvement revealed no significant intergroup difference at 2-month follow-up ( $p=0.84)$.

The secretory capacity of the meibomian gland was assessed by the MGYLS score. In the MiBoFlo group, the MGYLS score decreased from $8.68 \pm 3.64$ at baseline to $7.32 \pm 3.25$ at 1 month $(p<0.0001)$, and to $5.07 \pm 2.26$ at 2 months $(p<0.0001)$ vs. from $8.43 \pm 3.67$ at baseline to $5.15 \pm 3.30$ at 1 month $(p<0.0001)$, and to $2.98 \pm 2.78$ at 2 months

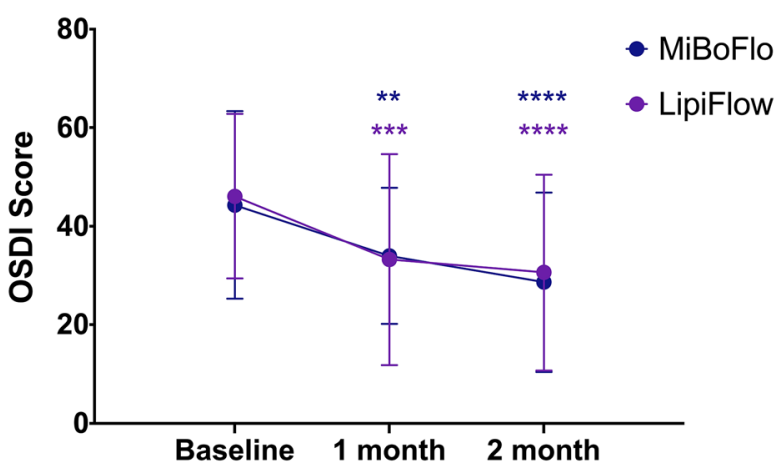

Fig. 1 Mean OSDI score of the MiBoFlo group and the LipiFlow group measured at baseline, 1 month, and 2 months. Asterisks indicate that values are significantly different from their respective baseline level $\left({ }^{* *} p<0.01\right.$; ${ }^{* * *} p<0.001{ }^{* * *} p<0.0001$ 


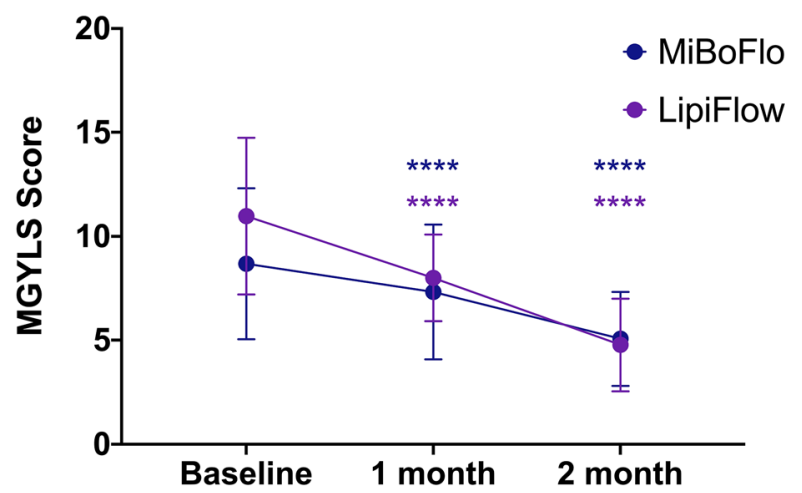

Fig. 2 Mean MGYLS score of the MiBoFlo group and the LipiFlow group measured at baseline, 1 month, and 2 months. ${ }^{* * * *}$ Values are significantly different from their respective baseline level $(p<0.0001)$

$(p<0.0001)$ in the LipiFlow group (Fig. 2). MGYLS improvement had no significant intergroup difference at 2-month follow-up $(p=0.09)$.

The meibum was assessed by MGS score. In the MiBoFlo group, the MGS score decreased from $9.20 \pm 3.70$ at baseline to $7.18 \pm 2.86$ at 1 month $(p>0.05)$, and to $4.86 \pm 2.18$ at 2 months $(p<0.001)$ vs. from $10.98 \pm 3.78$ at baseline to $8.00 \pm 2.09$ at 1 month $(p<0.001)$, and to $4.78 \pm 2.22$ at 2 months $(p<0.001)$ in the LipiFlow group (Fig. 3). MGS improvement did not show a significant intergroup difference at 2-month follow-up ( $p=0.13)$.

In the MiBoFlo group, the TMH changed from $0.23 \pm 0.08 \mathrm{~mm}$ at baseline to

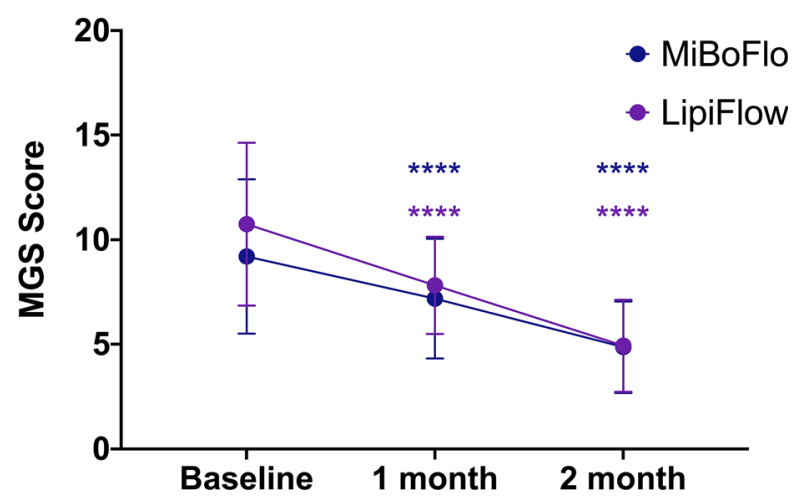

Fig. 3 Mean MGS score of the MiBoFlo group and the LipiFlow group measured at baseline, 1 month, and 2 months. ${ }^{* * * *}$ Values are significantly different from their respective baseline level $(p<0.0001)$
$0.24 \pm 0.07 \mathrm{~mm}$ at 1 month $(p=0.21)$, and to $0.27 \pm 0.13 \mathrm{~mm}$ at 2 months $(p=0.09)$ vs. from $0.25 \pm 0.12 \mathrm{~mm}$ at baseline to $0.24 \pm 0.10 \mathrm{~mm}$ at 1 month $(p=0.90)$, and to $0.24 \pm 0.08 \mathrm{~mm}$ at 2 months $(p>0.99)$ in the LipiFlow group.

In the MiBoFlo group, the NIKBUT changed from $6.44 \pm 3.46 \mathrm{~s}$ at baseline to $6.44 \pm 2.13 \mathrm{~s}$ at 1 month $(p>0.99)$, and to $5.99 \pm 2.67 \mathrm{~s}$ at 2 months $(p=0.79)$ vs. from $5.78 \pm 2.95 \mathrm{~s}$ at baseline to $5.33 \pm 2.62 \mathrm{~s}$ at 1 month $(p=0.81)$, and to $5.86 \pm 2.69 \mathrm{~s}$ at 2 months $(p>0.99)$ in the LipiFlow group.

CFS was used for assessing corneal epithelium. In the MiBoFlo group, the CFS score decreased from $0.16 \pm 0.43$ at baseline to $0.05 \pm 0.21$ at 1 month $(p=0.07)$, and to $0.02 \pm 0.15$ at 2 months $(p=0.10)$ vs. from $0.38 \pm 0.63$ at baseline to $0.30 \pm 0.56$ at 1 month $(p>0.99)$, and to $0.18 \pm 0.50$ at 2 months $(p=0.48)$ in the LipiFlow group.

The area of meibomian glands loss was evaluated by Meibo-Scan and ImageJ software. In the MiBoFlo group, the area of meibomian glands loss changed from $0.27 \pm 0.17$ at baseline to $0.27 \pm 0.18$ at 2 months $(p>0.05)$ vs. from $0.22 \pm 0.11$ at baseline to $0.22 \pm 0.10$ at 2 months $(p>0.05)$ in the LipiFlow group.

No subject in either group experienced any device-related adverse events that involved changes in VA, IOP, anterior segment, and facial skin. Only one case in the LipiFlow group encountered difficulties in the process of installing the eye cup because of a small palpebral fissure.

\section{DISCUSSION}

Currently, the therapeutic effect and maintenance time of LipiFlow are relatively clear, yet, there are few studies on the efficacy of MiBoFlo. The purpose of the present study was to explore the short-term therapeutic effects of MiBoFlo on patients with MGD in China.

The OSDI score observed in both groups was significantly improved at the first visit. There was no significant change in the 2-month follow-up; however, compared with the baseline level, the changes were of clinically significant. 
Miller et al. recruited 310 subjects in order to assess the minimal clinically important difference (MCID) for the OSDI, which is defined as "the smallest difference in score in that domain of interest which subjects perceive as beneficial and which would mandate, in the absence of troublesome side effects and excessive cost, a change in the patient's management" [17]. They found that the MCID ranged from 4.5 to 7.3 for mild or moderate disease and from 7.3 to 13.4 for severe disease [18], which suggested that the response to treatment among patients with severe symptoms should be set higher than the response to treatment among patients with moderate symptoms. Thus, in our study, by calculating the difference of OSDI score between baseline and 2-month follow-up for each patient, we found that in the MiBoFlo group, the mean improvement for severe patients was 11.3, and for mild to moderate patients it was 5.5; while in the LipiFlow group, the mean improvement for severe patients was 10.7, and for mild to moderate patients it was 5.1. This indicated that both devices were meaningful for the improvement of unpleasant symptoms in patients with DED.

Interestingly, as a result of the separation of dry eye symptoms and signs [19], the patient's "severe" OSDI score cannot indicate whether they are a patient with severe dry eye. The severity of DED should be comprehensively evaluated with symptoms and signs.

On the basis of the pathogenesis, observing the orifice and meibum is the easiest and most direct way for objective evaluation of signs. Thus, MGYLS and MGS were chosen as primary parameters for meibomian gland assessment. Our results showed that the MGYLS and MGS observed in the MiBoFlo group were significantly improved at the first and second month follow-up visit. It is worth noting that the results in the LipiFlow group revealed a more obvious trend of continued improvement in the 1-month follow-up period.

The comparison of the two groups suggested that the LipiFlow treatment resembled a "onestep process" more, while the MiBoFlo treatment seemed to be more "layer by layer". The following reasons could explain the difference in treatment effects between the two groups.
First of all, although both MiBoFlo and LipiFlow achieve the therapeutic effect by transferring heat from the device to the meibomian glands, the difference is the actual temperature at the meibomian glands. As MiBoFlo is an external eyelid-warming device, the heat must pass through the eyelid tissue to reach the meibomian glands. Overheating is not advisable in order to ensure the safety of the skin and cornea. Thus, heat loss is inevitable. LipiFlow is an internal eyelid-warming device designed to rest on the sclera and to transfer heat directly to the palpebral conjunctiva. In their research, Kenrick and Alloo reported that the upper palpebral conjunctival temperatures had increased from 37.0 to $42.0^{\circ} \mathrm{C}$ after 12 -min LipiFlow treatment; still, after $10 \mathrm{~min}$ of MiBoFlo treatment, the temperatures increased minimally [12].

Secondly, the pressure applied to the meibomian gland also tends to differ. The pressure of a deliberate blink is about 0.3 psi. As for patients with MGD, Korb and Blackie reported that the pressure required to obtain the nonliquid meibum varied from 5 to $40 \mathrm{psi}$, leading to moderate to significant discomfort [20]. During LipiFlow treatment, the eyelids were compressed between the bladder and the lid warmer at 6 psi simultaneously combined with the inserted eye cup. In the MiBoFlo group, the meibum is artificially discharged by gently massaging the outer eyelid during treatment, which is followed by eyelid compression, while the applied pressure is human controlled.

Summing up the aforementioned discussion, we hypothesize that LipiFlow covers and squeezes the lower $2 / 3$ of meibomian glands while uniformly heating, thus having the ability to empty abnormal meibum at once. Also, in the MiBoFlo group, the result tended to be the superimposed effect of multiple treatments. The clearing of the obstruction might restore individual glands to a more normal state, manifesting improvement of MGYLS and MGS. The smooth discharge of normal meibum is beneficial to the recovery of tear film homeostasis. Enhanced lubrication from improved aqueous retention leads to epithelial cell regeneration. Thus, following the improvement in tear film stability, the other objective signs would get 
better, such as TMH, NIKBUT, and CFS in the present study.

In addition, we observed that the improvement in CFS score in the MiBoFlo group was more obvious than in the LipiFlow group, which may be due to the small palpebral fissure in Chinese that may cause damage to the ocular surface during LipiFlow treatment.

In this study, ImageJ was used for analyzing the area of MG loss in the two groups. Similar to evaluating MGYLS and MGS scores, all MGs of the lower eyelids were analyzed, and not just the central MGs, because of the wide range of used massages. In the final analysis, no significant difference was found in both groups before and after treatment. However, in the study by Hura et al., it was indicated that the meibomian gland structure might increase after LipiFlow treatment relative to untreated controls [21]. The discrepancy between the two studies could be related to the follow-up time and evaluation methods.

No subject in either group experienced any device-related adverse events that involved changes in VA, IOP, anterior segment, and facial skin throughout the treatment. Only one case in the LipiFlow group encountered difficulties during the process of installing the eye cup because of a small palpebral fissure.

Such improvements in subjective symptoms and tear film stability following a painless, relaxing, and safe therapy stand in contrast to the most common treatments for patients with DED currently in use, such as pharmacotherapy. Pharmacological approaches require patient's adherence to a dosing regimen that typically involves single or multiple daily doses, often for extended periods of time. Considering that the aqueous supplementation had little effect on the treatment efficacy, we allowed patients to continue to use the same eye drops as before; however, the medications remained unchanged during the follow-up.

This was a preliminary study and, as such, it has some limitations. Firstly, in this study, it was difficult for us to mask patients and operators, so only the observers could be masked in order to avoid bias as much as possible. Secondly, an insufficient number of enrolled patients and follow-up time might cause deviations in the results. In the future clinical management of MGD, clinicians might consider adopting multiple-dose MiBoFlo therapy or combination therapy with LipiFlow.

\section{CONCLUSION}

As a portable and comfortable externally applied heat source, MiBoFlo treatment can advance treatment of MGD and provide at least 2 months of sustained improvement in both symptoms and meibomian gland function. We believe that MiboFlo could be used as a treatment option for patients with MGD or as a combined treatment option with the thermal pulsation system treatment.

\section{ACKNOWLEDGMENTS}

Funding. This study or publication or rapid service fee of this article was funded by "The Youth Beijing Scholars program", Natural Science Foundation of China (8217040526), and the Beijing Advanced Innovation Center for Big Data-Based Precision Medicine, Beijing Tongren Hospital, Beihang University \& Capital Medical University (BHTR-KFJJ-202001).

Authorship. All named authors meet the International Committee of Medical Journal Editors (ICMJE) criteria for authorship for this article, take responsibility for the integrity of the work as a whole, and have given their approval for this version to be published.

Authors' Contributions. Ying Jie and Lei Tian are the main persons in charge of this clinical trial and responsible for subjects enrolled. Siyuan Li and Ke Yang are responsible for designing the trial. Siyuan Li, Jingyi Wang, Lei Zhu and Jun Feng are responsible for the treatment and follow-up of the trial. Siyuan $\mathrm{Li}$ and Ke Yang wrote the main manuscript text. All authors reviewed the manuscript. 
Disclosures. Siyuan Li, Ke Yang, Jingyi Wang, Shang Li, Lei Zhu, Jun Feng, Lei Tian and Ying Jie have nothing to disclose.

Compliance with Ethics Guidelines. This study conformed to the principles of the Declaration of Helsinki. The study protocol was approved by the Ethics Review Committee of Beijing Tongren Hospital. The trial was registered at www.clinicaltrials.gov as NCT04310969. All patients were given a full explanation of the study, and written informed consent was obtained from all participants.

Data Availability. The datasets generated during and analyzed during the current study are not publicly available due to the need for further research but are available from the corresponding author on reasonable request.

Open Access. This article is licensed under a Creative Commons Attribution-NonCommercial 4.0 International License, which permits any non-commercial use, sharing, adaptation, distribution and reproduction in any medium or format, as long as you give appropriate credit to the original author(s) and the source, provide a link to the Creative Commons licence, and indicate if changes were made. The images or other third party material in this article are included in the article's Creative Commons licence, unless indicated otherwise in a credit line to the material. If material is not included in the article's Creative Commons licence and your intended use is not permitted by statutory regulation or exceeds the permitted use, you will need to obtain permission directly from the copyright holder. To view a copy of this licence, visit http://creativecommons.org/licenses/by$\mathrm{nc} / 4.0 /$.

\section{REFERENCES}

1. Jie Y, Xu L, Wu YY, Jonas JB. Prevalence of dry eye among adult Chinese in the Beijing Eye Study. Eye (Lond). 2009;23(3):688-93. https://doi.org/10. $1038 /$ sj.eye.6703101.
2. Stapleton F, Alves M, Bunya VY, et al. TFOS DEWS II epidemiology report. Ocul Surf. 2017;15(3):334-65. https://doi.org/10.1016/j.jtos.2017.05.003.

3. Craig JP, Nichols KK, Akpek EK, et al. TFOS DEWS II definition and classification report. Ocul Surf. 2017;15(3):276-83. https://doi.org/10.1016/j.jtos. 2017.05.008.

4. Sim HS, Petznick A, Barbier S, et al. A randomized, controlled treatment trial of eyelid-warming therapies in meibomian gland dysfunction. Ophthalmol Ther. 2014;3(1-2):37-48. https://doi.org/10.1007/ s40123-014-0025-8.

5. Teo CHY, Ong HS, Liu YC, Tong L. Meibomian gland dysfunction is the primary determinant of dry eye symptoms: analysis of 2346 patients. Ocul Surf. 2020;18(4):604-12. https://doi.org/10.1016/j. jtos.2020.06.008.

6. Geerling G, Tauber J, Baudouin C, et al. The international workshop on meibomian gland dysfunction: report of the subcommittee on management and treatment of meibomian gland dysfunction. Invest Ophthalmol Vis Sci. 2011;52(4):2050-64. https://doi.org/10.1167/iovs.10-6997g.

7. Murakami DK, Blackie CA, Korb DR. All warm compresses are not equally efficacious. Optom Vis Sci. 2015;92(9):e327-33.

8. Friedland BR, Fleming CP, Blackie CA, Korb DR. A novel thermodynamic treatment for meibomian gland dysfunction. Curr Eye Res. 2011;36(2):79-87. https://doi.org/10.3109/02713683.2010.509529.

9. Korb DR, Blackie CA. Restoration of meibomian gland functionality with novel thermodynamic treatment device-a case report. Cornea. 2010;29(8): 930-3.

10. Blackie CA, Coleman CA, Holland EJ. The sustained effect (12 months) of a single-dose vectored thermal pulsation procedure for meibomian gland dysfunction and evaporative dry eye. Clin Ophthalmol. 2016;10:1385-96. https://doi.org/10.2147/ OPTH.S109663.

11. Borchman D. The optimum temperature for the heat therapy for meibomian gland dysfunction. Ocul Surf. 2019;17(2):360-4. https://doi.org/10. 1016/j.jtos.2019.02.005.

12. Kenrick CJ, Alloo SS. The limitation of applying heat to the external lid surface: a case of recalcitrant meibomian gland dysfunction. Case Rep Ophthalmol. 2017;8(1):7-12. https://doi.org/10.1159/ 000455087.

13. Wolffsohn JS, Arita R, Chalmers R, et al. TFOS DEWS II diagnostic methodology report. Ocul Surf. 
2017;15(3):539-74. https://doi.org/10.1016/j.jtos. 2017.05.001.

14. Rose-Nussbaumer J, Lietman TM, Shiboski CH, et al. Inter-grader agreement of the ocular staining score in the Sjogren's International Clinical Collaborative Alliance (SICCA) registry. Am J Ophthalmol. 2015;160(6):1150-1153.e3. https://doi.org/10. 1016/j.ajo.2015.08.021.

15. Pult H, Riede-Pult B. Comparison of subjective grading and objective assessment in meibography. Cont Lens Anterior Eye. 2013;36(1):22-7. https:// doi.org/10.1016/j.clae.2012.10.074.

16. Greiner JV. Long-term (3 year) effects of a single thermal pulsation system treatment on meibomian gland function and dry eye symptoms. Eye Contact Lens. 2016;42(2):99-107. https://doi.org/10.1097/ ICL.0000000000000166.

17. Jaeschke R, Singer J, Guyatt GH. Measurement of health status: ascertaining the minimal clinically important difference. Control Clin Trials. 1989;10: 407-15.
18. Miller KL, Walt JG, Mink DR, et al. Minimal clinically important difference for the ocular surface disease index. Arch Ophthalmol. 2010;128(1): 94-101.

19. Vehof J, Sillevis Smitt-Kamminga N, Nibourg SA, Hammond CJ. Predictors of discordance between symptoms and signs in dry eye disease. Ophthalmology. 2017;124(3):280-6. https://doi.org/10. 1016/j.ophtha.2016.11.008.

20. Korb DR, Blackie CA. Meibomian gland therapeutic expression: quantifying the applied pressure and the limitation of resulting pain. Eye Contact Lens. 2011;37(5):298-301. https://doi.org/10.1097/ICL. 0b013e31821bc7c5.

21. Hura AS, Epitropoulos AT, Czyz CN, Rosenberg ED. Visible meibomian gland structure increases after vectored thermal pulsation treatment in dry eye disease patients with meibomian gland dysfunction. Clin Ophthalmol. 2020;14:4287-96. https:// doi.org/10.2147/OPTH.S282081. 